\title{
A covalent grafting approach for improving the dispersion of carbon black in styrene-butadiene rubber composites by copolymerizing $p$-(2,2'-diphenylethyl)styrene with a thermally decomposed triphenylethane pendant
}

Minglu Huang, Jianmin Lu*, Bingyong Han*, Ming Qiu, and Liqun Zhang,

State Key Laboratory of Chemical Resource Engineering, Beijing University of Chemical Technology, Beijing, 100029, P. R. China.

*Corresponding author:

J. Lu (lujm@mail.buct.edu.cn) and B.Han (hanby@mail.buct.edu.cn) 
Table S1. Recipes for the anionic polymerization of SBDR

\begin{tabular}{cccc}
\hline ingredients & SBDR -0 & SBDR -2 & SBDR -5 \\
\hline Butadiene/g & 75 & 75 & 75 \\
Styrene/g & 25 & 23 & 20 \\
DPES/g & 0 & 2 & 5 \\
Cyclohexane/L & 1 & 1 & 1 \\
TMEDA $/ \mathrm{mmol}$ & 0.15 & 0.15 & 0.15 \\
$n$-BuLi/mmol & 0.3 & 0.3 & 0.3 \\
\hline
\end{tabular}

Table S2. Recipes for preparing the CB filled rubber composites

\begin{tabular}{cc}
\hline Ingredients & Loading $\left(\mathrm{phr}^{\mathrm{a}}\right)$ \\
\hline SBDR & 100 \\
Carbon black (N330) & 40 \\
Zinc oxide & 5 \\
Stearic acid & 2 \\
Antioxidant $^{\mathrm{b}}$ & 1 \\
Accelerator $\mathrm{CZ}^{\mathrm{c}}$ & 1.51 \\
Sulfur & 1.7 \\
\hline
\end{tabular}

${ }^{\mathrm{a}}$ Weight in parts per 100parts rubber.

${ }^{\mathrm{b}} \mathrm{N}$-phenyl-1-naphthylamine.

${ }^{\mathrm{c}} \mathrm{N}$-cyclohexyl-2-benzothiazolylsulfenamide. 
Table S3. G ${ }_{0}$, $G_{\infty}$ ', and $G_{\infty}{ }^{\prime}-G_{0}$ ' of CB/SBDR vulcanizates with different DPES contents

\begin{tabular}{lccc}
\hline Samples & $\begin{array}{c}\mathrm{G}_{0}{ }^{\prime} \\
(\mathrm{KPa})\end{array}$ & $\begin{array}{c}\mathrm{G}_{\infty}{ }^{\prime} \\
(\mathrm{KPa})\end{array}$ & $\begin{array}{c}\mathrm{G}_{\infty}{ }^{\prime}-\mathrm{G}_{0}{ }^{\prime} \\
(\mathrm{KPa})\end{array}$ \\
\hline $\mathrm{CB} / \mathrm{SBDR}-0$ & 860 & 34 & 826 \\
$\mathrm{CB} / \mathrm{SBDR}-2$ & 709 & 40 & 669 \\
$\mathrm{CB} / \mathrm{SBDR}-5$ & 601 & 33 & 568 \\
\hline
\end{tabular}

Table S4. Mechanical properties of CB/SBDR vulcanizates with different DPES contents

\begin{tabular}{cccc}
\hline Samples & $\begin{array}{c}\text { Tensile strength } \\
(\mathrm{MPa})\end{array}$ & $\begin{array}{c}\text { Elongation at break } \\
(\%)\end{array}$ \\
\hline $\mathrm{CB} / \mathrm{SBDR}-0$ & 15.2 & 315.7 \\
$\mathrm{CB} / \mathrm{SBDR}-2$ & 18.2 & 317.9 \\
$\mathrm{CB} / \mathrm{SBDR}-5$ & 21.9 & 352.3 \\
\hline
\end{tabular}


Table S5. Variation of dynamic loss coefficient $(\tan \delta)$ with temperature at $0.02 \%$ strain and $1 \mathrm{~Hz}$ for CB/SBDR vulcanizates with different DPES contents.

\begin{tabular}{rlll}
\hline Samples & $\begin{array}{c}\text { DPES content } \\
\text { (wt \%) }\end{array}$ & \multicolumn{2}{c}{$\tan \delta$} \\
$\left(0{ }^{\circ} \mathrm{C}\right)$ & $\begin{array}{c}\tan \delta \\
\left(60{ }^{\circ} \mathrm{C}\right)\end{array}$ \\
\hline $\mathrm{CB} / \mathrm{SBDR}-0$ & 0 & 0.79 & 0.12 \\
$\mathrm{CB} / \mathrm{SBDR}-2$ & 1.9 & 0.87 & 0.11 \\
$\mathrm{CB} / \mathrm{SBDR}-5$ & 5.1 & 0.92 & 0.08 \\
\hline
\end{tabular}

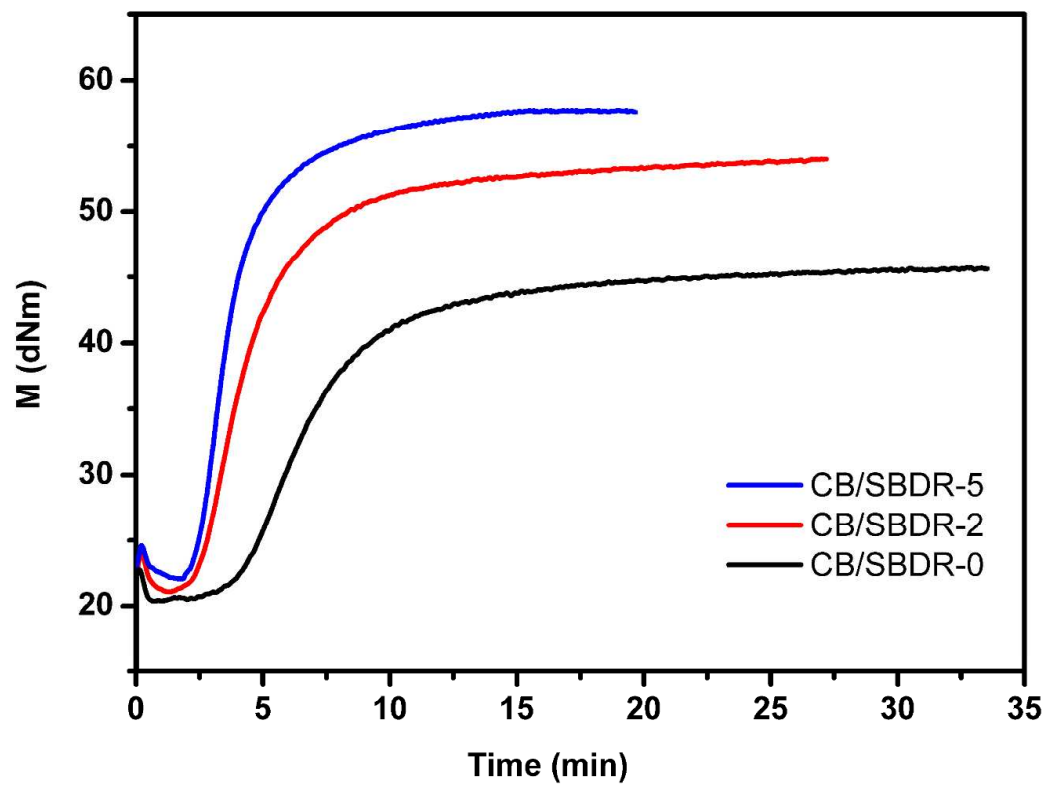

Figure S1. Vulcanization curve of CB/SBDR compounds. 